Proceedings of SALT 29: 706-726, 2019

\title{
Homogeneity or implicature: An experimental investigation of free choice*
}

\author{
Lyn Tieu \\ Western Sydney University \\ Jacopo Romoli \\ Ulster University
}

Cory Bill

Leibniz-ZAS

\begin{abstract}
A sentence containing disjunction in the scope of a possibility modal, such as Angie is allowed to buy the boat or the car, gives rise to the FREE CHOICE inference that Angie can freely choose between the two. This inference poses a well-known puzzle, in that it is not predicted by a standard treatment of modals and disjunction (e.g., Kamp 1974). To complicate things further, FREE CHOICE tends to disappear under negation: Angie is not allowed to buy the boat or the car doesn't merely convey the negation of free choice, but rather the stronger DUAL PROHIBITION reading that Angie cannot buy either one. There are two main approaches to the FREE CHOICE-DUAL PROHIBITION pattern in the literature. While they both capture the relevant data points, they make a testable, divergent prediction regarding the status of positive and negative sentences in a context in which Angie can only buy one of the two objects, e.g., the boat. In particular, the implicaturebased approach (e.g., Fox 2007; Klinedinst 2007; Bar-Lev \& Fox 2017) predicts that the positive sentence is true in such a context, but associated with a false implicature, while it predicts the negative sentence to be straightforwardly false. The alternative approach (e.g., Aloni 2018; Goldstein 2018; Willer 2017) predicts both the positive and negative sentences to be equally undefined. Investigating the contrast between these sentences in such a context therefore provides a clear way to address the debate between implicature and non-implicature accounts of FREE CHOICE. We present an experiment aiming to do just this, the results of which present a challenge for the implicature approach. We further discuss how the implicature approach could in theory be developed to account for our results, based on a recent proposal by Enguehard \& Chemla (2018) on the distribution of implicatures.
\end{abstract}

* For helpful feedback and discussion, we thank Amir Anvari, Moysh Bar-Lev, Richard Breheny, Ivano Caponigro, Lucas Champollion, Emmanuel Chemla, Gennaro Chierchia, Emile Enguehard, Danny Fox, Simon Goldstein, Martin Hackl, Nathan Klinedinst, Mora Maldonado, Matt Mandelkern, Paul Marty, Salvador Mascarenas, Paolo Santorio, Benjamin Spector, Yasu Sudo, Alexis Wellwood, and the audiences at XPRAG2019 in Edinburgh, SALT 29, and at MIT, NYU, UCSD, University of Göttingen, UCL, and University of Amsterdam. This research was supported in part by Western Sydney University through the University's Research Theme Champion support funding, and by the Leverhulme Trust grant RPG-2018-425.

(C2019 Tieu, Bill, and Romoli 
Homogeneity or implicature

Keywords: free choice, implicature, homogeneity, ternary task

\section{Introduction}

A sentence containing disjunction in the scope of a possibility modal, such as (1-a), gives rise to the so-called FREE CHOICE inference in (1-b), conveying that Angie is allowed to buy the boat and that she is allowed to buy the car. That is, she can freely choose between the two.

(1) a. Angie is allowed to buy the boat or the car.

b. $\rightsquigarrow$ Angie can choose between the two

FREE CHOICE

This inference poses a well-known puzzle, in that it is not predicted by standard treatments of modals and disjunction (see Kamp 1974 and much subsequent work, as well as Meyer 2018 for a detailed overview of the problem). To complicate things further, FREE CHOICE tends to disappear under negation: the corresponding negative sentence in (2-a) doesn't merely convey the negation of free choice; rather, it conveys the stronger DUAL PROHIBITION reading, conveying that Angie cannot buy either one.

(2) a. Angie is not allowed to buy the boat or the car.

b. $\rightsquigarrow$ Angie is not allowed to buy either one DUAL PROHIBITION

There are two main approaches to the FREE CHOICE-DUAL PROHIBITION pattern in the literature, one based on deriving FREE CHOICE as an implicature, the other encoding it in the meaning of disjunction and/or the modal. While both approaches can account for the basic cases above as well as a variety of more complex data, they differ with respect to a simple prediction regarding the status of sentences like (1-a) and (2-a) in a context in which Angie can only buy one of the two objects, e.g., the boat. In particular, the implicature approach predicts the positive case to be a true sentence with a false implicature, while it predicts the negative case to be straightforwardly false in such a context. The non-implicature approach, on the other hand, predicts both cases to be equally undefined. The comparison between these sentences in such a context therefore provides a simple way to address the debate between implicature and non-implicature approaches to FREE CHOICE.

We report an experiment aiming to test precisely these predictions. Specifically, we investigated the interpretation of sentences like (1-a) and (2-a), comparing them to positive and negative plain disjunctions like (3-a) and (4-a); the EXCLUSIVITY inference that arises from (3-a) (but not from its negation in (4-a)) is less controversially analyzed as an implicature. 
Tieu, Bill, and Romoli

(3) a. Angie bought the boat or the car.

b. $\rightsquigarrow$ Angie didn't buy both the boat and the car EXCLUSIVITY IMP

(4) a. Angie didn't buy the boat or the car.

b. $\rightsquigarrow$ Angie didn't buy either one NEGATED LITERAL MEANING

The results of our experiment revealed a clear difference between the two pairs of sentences $((1-a) /(2-a)$ and $(3-a) /(4-a))$ : while the latter gave rise to an asymmetric pattern, as predicted by the implicature approach, the former pair elicited the same response across polarities. As we discuss below, the similarity between the positive and negative free choice cases and the difference observed in the simple disjunction cases are challenging for the implicature approach. We outline the challenge in detail and briefly discuss how the implicature approach might be developed to account for our results, based on a recent proposal by Enguehard \& Chemla (2018) on the distribution of implicatures.

The rest of the paper is organized as follows. In Section 2, we provide further background on the FREE CHOICE-DUAL PROHIBITION pattern and outline the two approaches and their predictions. In Section 3, we briefly discuss some relevant previous studies. We present our experiment in Section 4, and discuss the implications of the findings for theories of free choice, including how the implicature approach might be further developed to account for the results. Section 5 concludes the paper.

\section{Background}

\subsection{Free choice}

As we have seen, a sentence like (1-a) and its negation in (2-a) pose a challenge for standard treatments of modals and disjunction. To illustrate the pattern more schematically, a configuration like (5-a) gives rise to the conjunctive FREE CHOICE inference in (5-b). Its negation in (6-a) also gives rise to a conjunctive DUAL PROHIBITION inference, which is stronger than the NEGATED FREE CHOICE inference (which would correspond to: $\neg \diamond \mathrm{B} \vee \neg \diamond \mathrm{C}$ ).

$$
\begin{array}{ll}
\text { a. } & \diamond(B \vee C) \\
\text { b. } & \diamond B \wedge \triangleright C \\
\text { a. } & \neg \diamond(B \vee C) \\
\text { b. } & \neg \diamond B \wedge \neg \triangleright C
\end{array}
$$

FREE CHOICE

DUAL PROHIBITION

In addition, the negated free choice reading re-emerges in cases like (7), in which a DUAL PROHIBITION reading of the first sentence would lead to a contradiction with the continuation. The coherence of (7) indicates that the first sentence is read with a negated free choice reading. 
Homogeneity or implicature

(7) Angie isn't allowed to buy the boat or the car. She's only allowed to buy the boat!

Relatedly, the FREE CHOICE inference can also be suspended in cases like (8), where a FREE CHOICE reading of the first sentence would lead to a contradiction with the second one. The coherence of (8) again tells us that the first sentence can be read as not entailing FREE CHOICE.

(8) Angie is allowed to buy the boat or the car. I don't remember which one.

Any approach to the FREE CHOICE-DUAL PROHIBITION pattern not only has to predict FREE CHOICE in the positive case and DUAL PROHIBITION in the negative one, but it also needs to account for the absence of such DUAL PROHIBITION and FREE CHOICE readings in cases like (7) and (8), respectively. ${ }^{1}$ We turn now to the two main approaches in the literature.

\subsection{Two approaches}

\subsubsection{The implicature approach}

The most prominent approach in the literature is based on a theory of implicatures and comes in different versions (e.g., Fox 2007; Klinedinst 2007; Chemla 2010; Franke 2011; Santorio \& Romoli 2017; Bar-Lev 2018; Bar-Lev \& Fox 2017). Without going into the details of the implementation, this account is based on three main ingredients: (i) a standard meaning for disjunction and possibility modals, (ii) an implicature-generating algorithm, which we can refer to as 'EXH', and (iii) a(n independently required) principle regulating the distribution of $\mathrm{EXH}$, which bans or strongly disfavors EXH under negation, as in (9) (see, among others, Chierchia, Fox \& Spector 2012 and Fox \& Spector 2018).

(9) Do not insert EXH in a sentence $S$ if the resulting meaning is weaker than that of $S$, unless forced to.

Theories differ in how they conceive of and define EXH, how many EXH operators one needs, and in which positions they must occur in order to generate FREE CHOICE. These differences will not be important for our purposes. What is relevant for us is that EXH gives rise to FREE CHOICE as an implicature.

To illustrate how this approach works, let us go back to the positive case in (1-a), repeated schematically below in (10).

1 The puzzle in fact extends more generally beyond the positive/negative dichotomy to upward- versus downward-entailing environments; see Fox (2007), among others, for discussion. 
Tieu, Bill, and Romoli

\begin{tabular}{|c|c|c|c|}
\hline & LITERAL MEANING & IMPLICATURE & RESULT \\
\hline POS & $\diamond(\mathrm{B} \vee \mathrm{C})=\diamond \mathrm{B} \vee \diamond \mathrm{C}$ & $\operatorname{EXH}(\diamond(B \vee C))=\diamond \mathrm{B} \wedge \diamond \mathrm{C}$ & $\diamond \mathrm{B} \wedge \triangleright \mathrm{C}$ \\
\hline NEG & $\neg \diamond(\mathrm{B} \vee \mathrm{C})=\neg \diamond \mathrm{B} \wedge \neg \vee \mathrm{C}$ & $* \neg(\mathrm{EXH}(\diamond(\mathrm{B} \vee \mathrm{C})))=\neg(\diamond \mathrm{B} \wedge \triangleright \mathrm{C})$ & $\neg \diamond \mathrm{B} \wedge \neg \vee \mathrm{C}$ \\
\hline
\end{tabular}

Table 1 Derivation of the FREE CHOICE-DUAL PROHIBITION pattern under the implicature account.

$$
\diamond(\mathrm{B} \vee \mathrm{C})=\diamond \mathrm{B} \vee \diamond \mathrm{C}
$$

LITERAL MEANING

The literal meaning of (10) does not entail FREE CHOICE; in fact it is simply equivalent to $\diamond \mathrm{B} \vee \diamond \mathrm{C}$ (there is at least one between the boat and the car that Angie is allowed to buy).

The weak meaning in (10), when negated, immediately gives rise to the strong DUAL PROHIBITION reading: (11) entails $\neg \diamond B \wedge \neg \diamond C$ (Angie is not allowed to buy the boat and she isn't allowed to buy the car).

$$
\neg[\diamond(\mathrm{B} \vee \mathrm{C})]=\neg \diamond \mathrm{B} \wedge \neg \diamond \mathrm{C}
$$

DUAL PROHIBITION

To generate FREE CHOICE, EXH is added to the positive sentence, generating the desired inference as an implicature.

$$
\operatorname{EXH}[\diamond(B \vee C)]=\diamond \mathrm{B} \wedge \diamond \mathrm{C}
$$

FREE CHOICE

Moreover, the principle in (9) prevents, or makes it very hard for EXH to appear in the scope of negation, which would otherwise give rise to a NEGATED FREE CHOICE reading, (13), rather than the stronger DUAL PROHIBITION above in (11).

$$
* \neg[\operatorname{EXH}[\diamond(\mathrm{B} \vee \mathrm{C})]]=\neg \diamond \mathrm{B} \vee \neg \diamond \mathrm{C}
$$

NEGATED FREE CHOICE

The derivations of the different meanings are schematized in Table 1.

Finally, the implicature approach can also account for the absence of FREE CHOICE and DUAL PROHIBITION in cases like (7) and (8). The latter can simply be a case in which EXH is not added to (10), giving rise to the weaker literal meaning that does not entail FREE CHOICE. This meaning is compatible with the continuation, where the speaker is explicit about being ignorant as to which of the boat and the car Angie is allowed to buy. (7) would be a case in which EXH does appear under negation (in the formulation of (9), EXH is allowed to appear under negation even if it leads to weakening, when this is 'forced' by the context). In this case, the potentially contradictory continuation would force EXH to appear under negation, giving rise to the NEGATED FREE CHOICE meaning in (13), which is compatible with the rest of the sentence and can therefore account for the felicity of (7). 
Homogeneity or implicature

In sum, given standard assumptions about the meanings of modals and disjunction, along with a theory of implicatures and a principle that regulates their distribution, the implicature approach can account for the FREE CHOICE-DUAL PROHIBITION pattern as well as cases in which these readings are absent. We turn now to the alternative, non-implicature approach.

\subsubsection{The non-implicature approach}

The implicature approach is not the only theoretical option available for explaining the FREE CHOICE-DUAL PROHIBITION pattern (see Zimmerman 2000, among others). Recently, a variety of alternative accounts have been proposed (e.g., Starr 2016; Aloni 2018; Willer 2017; Goldstein 2018; Rothschild \& Yablo 2018; Fusco 2015). For concreteness, we focus on the HOMOGENEITY account in Goldstein (2018) (see Rothschild \& Yablo 2018 for a similar proposal); as far as we can see, however, the main points below apply to any non-implicature approach.

The HOMOGENEITY account is based on four ingredients: (i) a strong meaning for sentences like (1-a), which directly asserts FREE CHOICE, (ii) a homogeneity presupposition requiring that either all alternatives are possible or none of them are, (iii) an operator that has the effect of cancelling the strong FREE CHOICE meaning, and (iv) another operator that can suspend DUAL PROHIBITION.

To illustrate, consider the positive case in (14-a). Given (i), FREE CHOICE is directly entailed. ${ }^{2}$ In addition, (14-a) also presupposes HOMOGENEITY, as in (14-b) (which in this case is entailed by the asserted FREE CHOICE meaning).

$$
\begin{array}{ll}
\text { a. } & \diamond(B \vee C)=\diamond B \wedge \diamond C \\
\text { b. } & \diamond B \leftrightarrow \nabla C
\end{array}
$$

FREE CHOICE HOMOGENEITY

While this approach directly captures FREE CHOICE by encoding it in the meaning of the positive sentence, it fails to immediately account for DUAL PROHIBITION. This is because in the negative case in (15-a), the asserted meaning is now simply the negation of FREE CHOICE. However, the latter, in combination with the HOMOGENEITY presupposition in (15-b) (which projects through negation), gives rise to the desired DUAL PROHIBITION reading in (15-c).

$$
\begin{array}{ll}
\text { a. } & \neg \diamond(B \vee C)=\neg(\diamond B \wedge \triangleright C) \\
\text { b. } & \nabla B \leftrightarrow \triangleright C \\
\text { c. } & \neg \diamond B \wedge \neg \vee C
\end{array}
$$

\section{NEGATED FREE CHOICE HOMOGENEITY DUAL PROHIBITION}

Finally, the HOMOGENEITY approach can capture examples like (7) and (8) by

2 See Goldstein (2018) for two possible ways of implementing this compositionally, by tweaking the standard meaning of modals or that of disjunction. 
Tieu, Bill, and Romoli

\begin{tabular}{c|c|c|c} 
& ASSERTED MEANING & PRESUPPOSED MEANING & RESULT \\
\hline POSITIVE & $\diamond(\mathrm{B} \vee \mathrm{C})=\diamond \mathrm{B} \wedge \triangleright \mathrm{C}$ & $\diamond \mathrm{B} \leftrightarrow \diamond \mathrm{C}$ & $\diamond \mathrm{B} \wedge \triangleright \mathrm{C}$ \\
\hline NEGATIVE & $\neg \diamond(\mathrm{B} \vee \mathrm{C})=\neg(\diamond \mathrm{B} \wedge \diamond \mathrm{C})$ & $\diamond \mathrm{B} \leftrightarrow \diamond \mathrm{C}$ & $\neg \diamond \mathrm{B} \wedge \neg \vee \mathrm{C}$ \\
\hline
\end{tabular}

Table 2 Derivation of the FREE CHOICE-DUAL PROHIBITION pattern under the HOMOGENEITY account.

introducing two additional mechanisms. The first is an operator '!' that has the effect of cancelling FREE CHOICE when merged below the modal. ${ }^{3}$

$$
\diamond(!(B \vee C))=\diamond B \vee \diamond C
$$

The second is a local accommodation operator ' $\mathscr{A}$ ' (invoked independently in the presupposition literature, see Beaver 2001 and Fox 2012, among others), which, when merged below negation, makes the homogeneity presupposition an entailment and prevents it from projecting. The resulting meaning is the weak one in (17).

$$
\begin{aligned}
& \neg[\mathscr{A}[\diamond(\mathrm{B} \vee C)]]= \\
& \neg[(\diamond \mathrm{B} \wedge \diamond \mathrm{C}) \wedge(\diamond \mathrm{B} \leftrightarrow \Delta \mathrm{C})]= \\
& \neg[(\diamond \mathrm{B} \wedge \triangleright \mathrm{C})]=\neg \diamond \mathrm{B} \vee \neg \diamond \mathrm{C}
\end{aligned}
$$

NEGATED FREE CHOICE

In sum, combining the asserted meaning and the homogeneity presupposition, the homogeneity approach can capture the basic pattern, as well as the suspension of the FREE CHOICE and DUAL PROHIBITION readings (through the use of two additional operators). Table 2 provides a schematic illustration of the derivations of the different meanings under this approach.

\subsubsection{Summary}

The two theoretical approaches we have described can account for the basic FREE CHOICE and DUAL PROHIBITION readings, as well as cases in which these readings appear to be absent. On the empirical end of things, a variety of more complex data points have been discussed in the literature, including free choice in the scope of universal and negative existential quantifiers (Chemla 2009; Van Tiel 2012; BarLev \& Fox 2017; Bar-Lev 2018), modified numerals (Gotzner, Romoli \& Santorio 2017, to appear), different modal bases (Nouwen to appear; Bar-Lev 2018), free choice items beyond disjunction (Chierchia 2013), the interaction between free choice and presuppositions (Romoli \& Santorio 2019; Marty \& Romoli 2019), and

3 The operator ! essentially has the effect of double negation; we again refer the reader to Goldstein (2018) for details. 
Homogeneity or implicature

the processing of free choice (Chemla \& Bott 2014) and its acquisition in young children (Tieu, Romoli, Zhou \& Crain 2016). The jury is still out on which of the two approaches sketched above can best account for the observed empirical landscape. ${ }^{4}$ In what follows, we will concentrate on a divergent prediction of the two approaches. ${ }^{5}$

\subsection{Predictions}

The two approaches we have described make similar predictions for the basic FREE CHOICE-DUAL PROHIBITION pattern, as well as for a variety of related data. There is one prediction where they diverge, however, which to our knowledge has been untested (although similar discussions exist in the context of plurals, see Križ 2015; Križ \& Chemla 2015; Tieu, Bill, Romoli \& Crain 2017a; Renans, Romoli, Makri, Tieu, de Vries, Folli \& Tsoulas 2018). This divergent prediction has to do with the status of the basic positive and negative cases. In particular, recall that under the implicature approach, FREE CHOICE arises as an implicature while DUAL PROHIBITION is simply a part of the literal meaning.

(1-a) Angie is allowed to buy the boat or the car. $\rightsquigarrow$ Angie can choose between the two

IMPLICATURE

(2-a) Angie is not allowed to buy the boat or the car. $\rightsquigarrow$ Angie cannot buy either one

LITERAL MEANING

Under the homogeneity approach, on the other hand, FREE CHOICE is part of the literal meaning, while DUAL PROHIBITION arises via the homogeneity presupposition. Crucially, under this approach, both the positive and negative cases are associated with the same homogeneity presupposition.

(1-a) Angie is allowed to buy the boat or the car. $\rightsquigarrow$ Angie can buy one iff she can buy the other

HOMOGENEITY

4 While the non-implicature approach can more straightforwardly account for certain observed differences between free choice and other implicatures, it is more stipulative in nature. In particular, while the implicature approach does not need any extra assumptions about the meanings of modals and disjunction, the non-implicature approach needs to tweak these meanings in particular ways (see Bar-Lev 2018, Aloni 2018, and Romoli \& Santorio 2019 for discussion).

5 A third option is the account in Barker (2010), which encodes FREE CHOICE in the semantics while deriving DUAL PROHIBITION as an implicature. This approach makes the opposite prediction of the standard implicature approach: in a context in which Angie is only allowed to buy the boat, a sentence like (1-a) is predicted to be false, while its negative counterpart in (2-a) is predicted to be an implicature violation. That is, this approach predicts an asymmetry between the positive and negative free choice cases, but in the opposite direction of the corresponding disjunction cases. As we will see below, our results are also challenging for this kind of implicature approach. 
Tieu, Bill, and Romoli

\begin{tabular}{l|l|l} 
& IMPLICATURE & HOMOGENEITY \\
\hline POSITIVE & IMPLICATURE VIOLATION & PRESUPPOSITION FAILURE \\
\hline NEGATIVE & FALSITY & PRESUPPOSITION FAILURE \\
\hline
\end{tabular}

Table 3 Predictions of the implicature and homogeneity approaches for sentences like (1-a) and (2-a), in contexts in which only one of the disjuncts is true.

(2-a) Angie is not allowed to buy the boat or the car.

$\rightsquigarrow$ Angie can buy one iff she can buy the other

HOMOGENEITY

The differences in the status of the positive and negative sentences can be brought out in a context in which only one of the disjuncts is true. To illustrate, consider a context in which Angie is only allowed to buy the boat. In this context, the homogeneity account predicts both the positive and negative cases to be undefined, as their presupposition is not satisfied. The implicature account, on the other hand, predicts a difference in status across the two polarities: it predicts the positive to be a literally true sentence, but with a false implicature, while it predicts the negative case to be plainly false. The predictions are summarized in Table 3.

To sharpen the intuitions, consider the comparison with the corresponding simple disjunction case: (3-a) gives rise to the exclusivity inference in (3-b), which disappears under negation in (4-a). In a context in which Angie bought both the boat and the car, (3-a) is predicted to be true but with a false implicature, while (4-a) is predicted to be plainly false.

a. Angie bought the boat or the car.

b. $\rightsquigarrow$ Angie didn't buy both the boat and the car EXCLUSIVITY IMP

a. Angie didn't buy the boat or the car.

b. $\quad \rightsquigarrow$ Angie didn't buy either one

NEGATED LITERAL MEANING

We can state the predictions as follows: the implicature approach predicts a similar pattern for the pairs in (1-a)/(2-a) and (3-a)/(4-a), reflecting a false implicature in the positive cases and a false literal meaning in the negative cases. The homogeneity account, on the other hand, is compatible with a difference between the pairs, in that (1-a) and (2-a), unlike (3-a) and (4-a), are predicted to have the same status (they are both predicted to be undefined). These predictions can be recast as in Table 4.

Quantitatively, observing a statistical interaction between Inference Type (EXCLUSIVITY vs. FREE CHOICE) and Polarity (positive vs. negative) would present a challenge for the implicature approach, but would be entirely in line with the ho- 
Homogeneity or implicature

\begin{tabular}{l|l|l|l}
\hline & DISJUNCTION & FREE CHOICE (IMP) & FREE CHOICE (HOM) \\
\hline POS & IMP VIOLATION & IMP VIOLATION & PRESUPPOSITION FAILURE \\
\hline NEG & FALSITY & FALSITY & PRESUPPOSITION FAILURE \\
\hline
\end{tabular}

Table 4 Predictions of the implicature and homogeneity approaches for sentences like (1-a)/(2-a) and (3-a)/(4-a), in contexts in which only one of the disjuncts is true.

mogeneity approach. Testing these predictions gives us a simple way to distinguish between the two theoretical approaches. Before turning to our experiment, we will briefly outline some previous studies that the experiment builds upon.

\section{Previous studies}

In recent years, a variety of studies have used a ternary judgment task to investigate implicatures (Katsos \& Bishop 2011; Tieu et al. 2017a; Renans et al. 2018), presuppositions (Abrusan \& Szendroi 2013), and homogeneity (Križ \& Chemla 2015; Tieu, Kriz \& Chemla 2019). As first conceived of in Katsos \& Bishop (2011) for implicatures, the idea behind the ternary judgment task is that the lowest valued judgment (e.g., the smallest reward) is reserved for false sentences, the highest valued judgment (e.g., the biggest reward) is reserved for true and felicitous sentences, and the intermediate judgment is meant for true but infelicitous sentences, e.g., sentences with a true literal meaning but a false implicature.

Tieu et al. (2017a) made use of such a ternary judgment task to investigate the comprehension of plural noun phrases, comparing the multiplicity inference of bare plurals to the exclusivity inference of disjunction. In their study, participants saw pictures of cartoon characters deciding what to buy at the store, and heard a puppet's guesses as to what would or wouldn't happen (the so-called 'prediction' mode of the judgment task; see Tieu, Yatsushiro, Cremers, Romoli, Sauerland \& Chemla 2017b, among others). Participants then saw the outcome and had to decide whether to reward the puppet with a small, medium, or large strawberry.

In particular, on the disjunction trials, participants heard positive and negative sentences such as (18) and (19), in contexts in which both disjuncts turned out to be true; for example, Tiger ended up buying both the apple and the banana, leaving behind a third object.

(18) Tiger will buy the apple or the banana.

(19) Tiger will not buy the apple or the banana. 
In the target context in which both disjuncts turn out to be true, the positive (18) is a true sentence with a false implicature, while its negative counterpart in (19) is plainly false. Participants were therefore expected to select the intermediate reward for (18), and the minimal reward corresponding to plain falsity for (19).

The results indeed revealed a clear contrast between plain falsity and implicature violation. Participants favored the intermediate reward for the positive targets, and gave mostly minimal rewards for the negative targets. This is in line with an implicature approach to the exclusivity inference, with participants mapping the intermediate reward to implicature violation and the minimal reward to falsity. This result is important because it provides a baseline against which to compare other phenomena that have also been subjected to an implicature analysis; indeed, Tieu et al. (2017a) compared the pattern of exclusivity inferences with that of multiplicity inferences in order to investigate the implicature approach to plurality. We will use the same logic in the present study, comparing the implicature of disjunction to the free choice inference.

\section{Experiment}

We used a $2 \times 2$ design with Inference Type (free choice vs. disjunction, between subjects) and Polarity (positive vs. negative, within subjects) as factors.

\subsection{Methods}

\subsubsection{Participants}

114 participants were recruited through Amazon Mechanical Turk and randomly assigned to the free choice (FC) or disjunction (OR) condition; three were excluded for not reporting English as a native language, leaving 111 participants for analysis (56 FC, 55 OR). Participants were paid \$.75 USD for the 5-minute study.

\subsection{Procedure}

Participants completed a ternary judgment task in prediction mode, implemented in Qualtrics. They had to read a puppet's guesses about what a character was allowed/not allowed to buy (FC condition) or about what a character bought/didn't buy (OR condition). Participants then had to decide upon seeing the outcome whether the puppet ought to receive a small strawberry (described in the instructions as corresponding to 'totally wrong' guesses), a large strawberry ('totally right'), or a medium-sized strawberry ('in between, not totally right but not totally wrong'). 


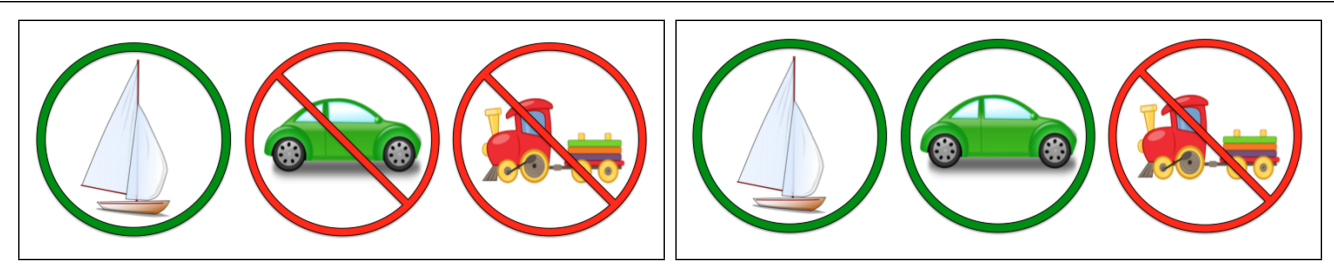

Figure 1 Example visual stimuli for FC and OR targets: the image on the left was paired with the positive and negative FC targets in (1-a) and (2-a); the image on the right was paired with the positive and negative OR targets in $(3-a)$ and (4-a).

Participants indicated their response by clicking on the button that had the desired reward on it.

\subsubsection{Materials}

Objects that were 'allowed' (FC condition) or bought (OR condition) were visually represented in a green circle; objects that were 'disallowed' (FC condition) or were not bought (OR condition) were represented with a red circle with a line across it.

In the FC condition, the critical positive and negative target sentences (e.g., (1-a) and (2-a), repeated below) were presented in contexts such as Figure 1 [left], in which only one of the disjuncts was 'allowed' (i.e. Angie was only allowed to buy the boat).

(1-a) Angie is allowed to buy the boat or the car.

(2-a) Angie is not allowed to buy the boat or the car.

In the OR condition, the positive and negative target sentences (e.g., (3-a) and (4-a), repeated below) were presented in contexts like Figure 1 [right], which made false the exclusivity inference (i.e. Angie bought both the boat and the car).

(3-a) Angie bought the boat or the car.

(4-a) Angie didn't buy the boat or the car.

In addition to the target items, participants received clearly true and clearly false (positive and negative) control items; see Figure 2 for the FC controls and Figure 3 for the OR controls.

In total, participants received 2 training items, followed by 8 targets ( 4 positive, 4 negative) and 8 controls ( 2 true and 2 false positive controls, 2 true and 2 false 


FASITIVE

Figure 2 Example visual stimuli for FC controls: (1-a) was paired with the topleft image to create a positive true control, and with the bottom-left image to create a positive false control. (2-a) was paired with the topright image to create a negative true control, and with the bottom-right image to create a negative false control.

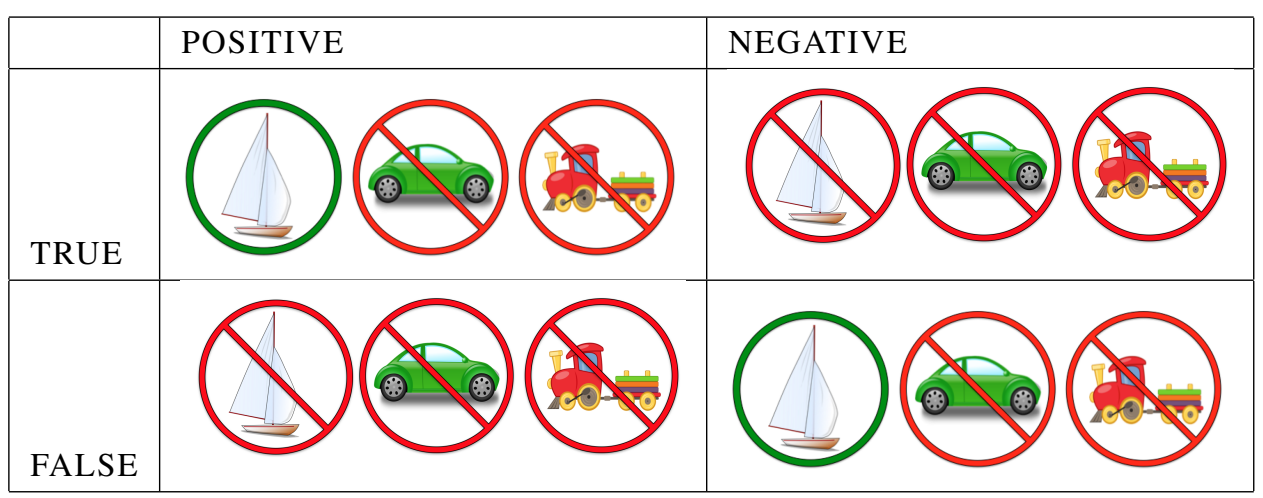

Figure 3 Example visual stimuli for OR controls: (3-a) was paired with the topleft image to create a positive true control, and with the bottom-left image to create a positive false control. (4-a) was paired with the topright image to create a negative true control, and with the bottom-right image to create a negative false control.

negative ones). Every picture/context contained three objects, to avoid any infelicity associated with using disjunction in a context with only two objects. ${ }^{6}$

6 See Skordos, Feiman, Bale \& Barner (2018) for discussion of potential infelicities associated with the use of disjunction in certain experimental contexts. 


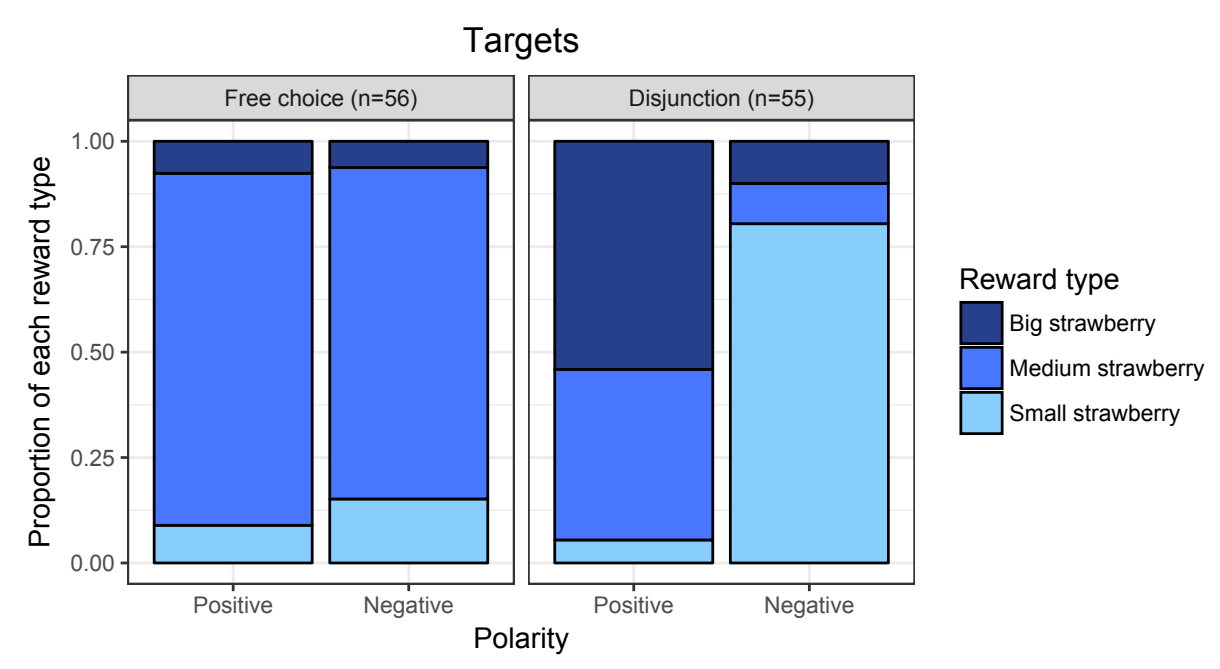

Figure 4 Proportion of each reward type selected in response to free choice and disjunction targets.

\subsection{Results}

As can be seen in Figure 4, participants primarily gave the intermediate reward in response to both positive and negative FC targets, while they gave an asymmetric pattern of responses to the positive and negative OR targets. We fitted a mixed effects cumulative link model to responses to the targets with Inference Type, Polarity, and their interaction as fixed effects, and random by-participant slopes for Polarity. Model comparisons between the maximal model and those without each of the fixed effects revealed a marginal effect of Inference Type $\left(\chi^{2}(1)=3.2, p=.07\right)$, a significant effect of Polarity $\left(\chi^{2}(1)=102, p<.001\right)$, and a significant interaction between Inference Type and Polarity $\left(\chi^{2}(1)=88, p<.001\right)$, with participants showing a greater difference between polarities for OR than for FC.

Figure 5 displays the results for the FC and OR controls. Participants' responses to the controls were mostly as expected, with maximal rewards for true controls and minimal rewards for false controls. In the disjunction condition, however, the positive true and negative false controls elicited a relatively large proportion of intermediate responses. These intermediate responses are surprising: in the positive case, the context was one in which Angie bought only the boat, thus (3-a) should have been judged as clearly true, and in the negative case, the same context was paired with (4-a), which therefore should have been judged as clearly false.

The intermediate responses in these cases could be related to participants' preferences regarding the scope of disjunction relative to negation, or to potential residual 


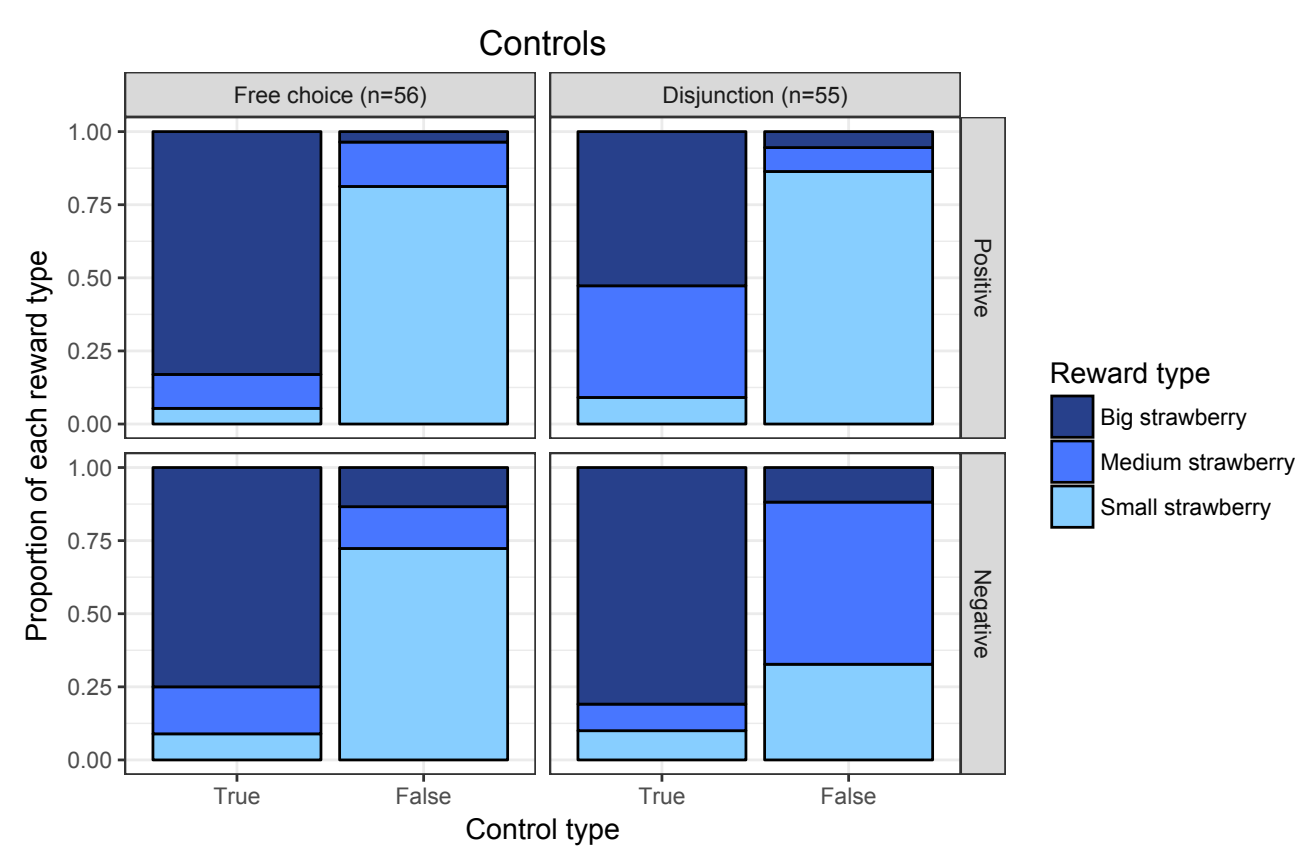

Figure 5 Proportion of each reward type selected in response to free choice and disjunction control items.

effects of the ignorance inference of disjunction (despite our best efforts to make the disjunction felicitous by presenting the sentences in prediction mode). These factors should be investigated more carefully in future research. What is relevant for present purposes is that participants treated the controls differently from the free choice targets, and so at least at first glance, whatever factors are playing a role in the former can't fully explain participants' performance on the latter.

\subsection{Discussion}

The observed difference between the free choice and disjunction targets, and in particular the observed interaction between Inference Type and Polarity, is challenging for the implicature account, which predicts a similar pattern for the two sentence types, across polarities. On the other hand, the results are straightforwardly in line with the homogeneity account, which predicts (1-a) and (2-a) to be equally undefined in the given context, and is compatible with the observed interaction between Inference Type and Polarity.

Note that a response to the challenge against the implicature account cannot lie (entirely) in a 'scalar diversity' effect (van Tiel, van Miltenburg, Zevakhina \& 
Homogeneity or implicature

Geurts 2016). That is, the observed interaction cannot be explained by appealing to a relative difference in the strength of the free choice and exclusivity inferences. This line of explanation would at most be able to account for the difference observed in the positive condition, but would be silent about the difference observed in the negative condition. ${ }^{7}$ We turn next to a promising direction that might be able to account for the negative condition.

\subsection{A possible solution to the challenge}

As mentioned, it is not clear how one might address the challenge to the implicature approach by appealing to the notion of scalar diversity, or to a difference in the alternatives involved in computing free choice and exclusivity. A possible alternative direction would be to reconsider the principle regulating the distribution of implicatures.

To illustrate, consider the standard principle in (9), which strongly disfavors the appearance of EXH in the scope of negation. As with our examples, the presence of EXH in the scope of negation weakens the meaning of the overall sentence and is thus blocked. Consider now what would happen if we were to lift the ban in (9): we could then parse the negative (2-a), repeated below, as in (20). The latter is associated with the weaker NEGATED FREE CHOICE meaning, $\neg \diamond C \vee \neg \diamond \mathrm{B}$, which is true in the given target context (Angie doesn't have free choice, as she can only buy the boat).

(2-a) Angie is not allowed to buy the boat or the car.

(20) not[EXH[Angie is allowed to buy the boat or the car]] $=\operatorname{not}[$ Angie can choose between the two]

NEGATED FC

If (20) were a viable option, then (2-a) could be associated with a true reading in the context (in addition to that in (21) without EXH, which would give rise to a false meaning instead, as seen above).

not[Angie is allowed to buy the boat or the car]

DUAL PROHIBITION

On the basis of this, one might hypothesize that the reason people gave intermediate responses to the negative FC targets was that the target sentences were associated with two different readings, one true and one false, resulting in an intermediate status (see Bill, Romoli \& Schwarz 2018 for a similar idea in relation to response times

7 The nature of the alternatives involved in inference computation has been argued to play a role in observed differences between free choice and other inferences, in processing and acquisition (Chemla \& Bott 2014; Tieu et al. 2016); however, it is not clear to us how appealing to alternatives would be able to account for the present data. 
associated with scalar implicatures and presuppositions).

The problem with this line of explanation is that it readily extends to the negative disjunction case in (4-a), repeated below. This is because the latter could also be analyzed as in (22), giving rise to a weaker meaning, $\neg \mathrm{B} \vee \neg C$, which is true in the given context.

(4-a) Angie didn't buy the boat or the car.

not[EXH[Angie bought the boat or the car]]

not[Angie bought the boat or the car but not both]

NEGATED EXCL

Therefore, simply abandoning the principle in (9) would not allow us to account for the difference between the free choice and disjunction conditions (not to mention the fact that it would leave us without an explanation for the distribution of implicatures). What's needed is to replace (9) with a principle that still disallows EXH under negation in cases like (4-a), but allows it in cases like (2-a). A recent proposal in the literature, Enguehard \& Chemla (2018), has independently argued for a principle that achieves exactly this. Enguehard \& Chemla (2018) argue that the standard formulation in (9) based on logical strength should be replaced by a constraint based on a notion of 'connectedness,' a logical notion related to monotonicity. Roughly, the principle in (23) makes a parse strongly dispreferred if it doesn't give rise to a connected meaning.

(23) Among the parses of a sentence (with or without EXH), those that result in non-connected meanings are dispreferred/marked.

Formal details aside (for which we refer the reader to Enguehard \& Chemla 2018), what is relevant here is that this proposal differs from the standard one precisely in that it predicts EXH not to be banned in the scope of negation with FREE CHOICE, while still predicting the standard asymmetry between positive and negative in the case of simple disjunction. ${ }^{8}$ Their argument is that it would intuitively be easier to force a NEGATED FREE CHOICE reading as in (24), than the corresponding negated exclusivity meaning in (25). ${ }^{9}$

8 This is the case only if an anti-conjunctive inference is not derived (see Enguehard \& Chemla 2018). 9 Both are actually possible with marked intonation, but the claim is that (24) is possible and relatively easy without such an intonation. As Enguehard \& Chemla (2018) discuss, their proposal doesn't account for why the negated free choice reading in (24), while possibly easier to access than the corresponding negated exclusivity reading, is intuitively still more difficult than the DUAL PROHIBITION reading in (i).

(i) Angie is not allowed to buy the boat or the car.

DUAL PROHIBITION 
Homogeneity or implicature

(24) Angie is not allowed to buy the boat or the car. She's only allowed to buy the boat!

(25) Angie didn't buy the boat or the car. She bought both!

The intuitive difference between (24) and (25) is in line with what we observed in our experiment, in particular with respect to the comparison between the negative free choice and disjunction conditions. Under this hypothesis, participants would have found it easier to read the negative FC targets with an embedded EXH than to do the same for the corresponding negative OR targets. As a result, the former had a true reading in the context, and consequently led participants to choose the medium strawberry. This proposal presents a promising direction for making the implicature approach compatible with our results (perhaps in combination with considerations of scalar diversity, to account for the difference in the positive conditions).

\section{Conclusion and further directions}

A sentence containing disjunction in the scope of a possibility modal, such as Angie is allowed to buy the boat or the car, gives rise to the FREE CHOICE inference that Angie can freely choose between the two. As is well known, this inference is puzzling for standard treatments of modals and disjunction (Kamp 1974). In addition, and to complicate things further, FREE CHOICE tends to disappear under negation: Angie is not allowed to buy the boat or the car doesn't merely convey the negation of free choice, but rather the stronger DUAL PROHIBITION reading that Angie cannot buy either one. There are two main approaches to this pattern in the literature, including one based on implicature. While both the implicature and non-implicature approaches cover the basic pattern and more complicated related data points, they diverge in what they predict for the status of positive and negative sentences in certain contexts. In this paper, we presented an experiment testing these predictions, the results of which present a challenge for the implicature approach but are more straightforwardly in line with the homogeneity approach. At a more general level, our results are consistent with previous findings in the literature of crucial differences between free choice and implicatures, e.g., in their processing and acquisition profiles (Chemla \& Bott 2014; Tieu et al. 2016).

One could take our results as providing support for a non-implicature approach; alternatively, the findings might encourage us to refine the implicature approach, by reconsidering, for instance, the principle regulating the distribution of the EXH operator. Regardless of the theoretical choice pursued, empirically investigating the relative status of the positive and negative sentences provides a useful way to address the debate between implicature and non-implicature approaches to free choice. Such comparative investigations may also prove fruitful for better understanding a variety 
Tieu, Bill, and Romoli

of other phenomena at the semantics-pragmatics interface.

\section{References}

Abrusan, Marta \& Kriszta Szendroi. 2013. Experimenting with the King of France. Semantics \& Pragmatics 6(10). 1-43.

Aloni, Maria. 2018. FC disjunction in state-based semantics. Ms., University of Amsterdam.

Bar-Lev, Moshe. 2018. Free choice, homogeneity and innocent inclusion: The Hebrew University of Jerusalem dissertation.

Bar-Lev, Moshe \& Danny Fox. 2017. Universal free choice and innocent inclusion. In Semantics and Linguistic Theory 27, 95-115.

Barker, Chris. 2010. Free choice permission as resource-sensitive reasoning. Semantics and Pragmatics 3(10). 1-38.

Beaver, David. 2001. Presupposition and Assertion in Dynamic Semantics. Stanford University: CSLI Publications.

Bill, Cory, Jacopo Romoli \& Florian Schwarz. 2018. Processing presuppositions and implicatures: Similarities and differences. Frontiers in Communication 3(44).

Chemla, Emmanuel. 2009. Universal implicatures and free choice effects: Experimental data. Semantics and Pragmatics 2(2).

Chemla, Emmanuel. 2010. Similarity: towards a unified account of scalar implicatures, free choice permission and presupposition projection. Ms., Ecole Normale Supérieure.

Chemla, Emmanuel \& Lewis Bott. 2014. Processing inferences at the semantics/pragmatics frontier: disjunctions and free choice. Cognition 130(3). 380396.

Chierchia, Gennaro. 2013. Logic in grammar: Polarity, free choice, and intervention. Oxford University Press.

Chierchia, Gennaro, Danny Fox \& Benjamin Spector. 2012. The grammatical view of scalar implicatures and the relationship between semantics and pragmatics. In Claudia Maienborn, Klaus von Heusinger \& Paul Portner (eds.), Semantics: An international handbook of natural language meaning volume 3, Berlin: Mouton de Gruyter.

Enguehard, Emile \& Emmanuel Chemla. 2018. Connectedness as a constraint on exhaustification. Ms., Ecole Normale Supérieure.

Fox, Danny. 2007. Free choice and the theory of scalar implicatures. In Uli Sauerland \& Penka Stateva (eds.), Presupposition and Implicature in Compositional Semantics, 71-120. Palgrave.

Fox, Danny. 2012. Presupposition projection from quantificational sentences: trivalence, local accommodation, and presupposition strengthening. In Ivano Capon- 
Homogeneity or implicature

igro \& Carlo Cecchetto (eds.), From grammar to meaning: The spontaneous logicality of language, 201-232. Cambridge University Press.

Fox, Danny \& Benjamin Spector. 2018. Economy and embedded exhaustification. Natural Language Semantics 26(1). 1-50.

Franke, Michael. 2011. Quantity implicatures, exhaustive interpretation, and rational conversation. Semantics and Pragmatics 4(1). 1-82. doi:10.3765/sp.4.1.

Fusco, Melissa. 2015. Deontic modality and the semantics of choice. Philosophers' Imprint 15.

Goldstein, Simon. 2018. Free choice and homogeneity. Ms., Lingnan University Hong Kong.

Gotzner, Nicole, Jacopo Romoli \& Paolo Santorio. 2017. More free choice and more inclusion: An experimental investigation of free choice in non-monotonic environments. Ms. Ulster University, ZAS, and UCSD.

Gotzner, Nicole, Jacopo Romoli \& Paolo Santorio. To appear. Choice and prohibition in non-monotonic contexsts. Natural Language Semantics.

Kamp, Hans. 1974. Free choice permission. The Aristotelian Society 74. 57-74.

Katsos, Napoleon \& Dorothy VM Bishop. 2011. Pragmatic tolerance: Implications for the acquisition of informativeness and implicature. Cognition 120(1). 67-81.

Klinedinst, Nathan. 2007. Plurality and possibility: UCLA dissertation.

Križ, Manuel. 2015. Aspects of homogeneity in the semantics of natural language: University of Vienna dissertation.

Križ, Manuel \& Emmanuel Chemla. 2015. Two methods to find truth-value gaps and their application to the projection problem of homogeneity. Natural Language Semantics 23. 205-248.

Marty, Paul \& Jacopo Romoli. 2019. Presupposed free choice and the theory of scalar implicatures. Ms., ZAS and Ulster University.

Meyer, Marie-Christine. 2018. An apple or a pear: free choice disjunction. In H. Rullmann T.E. Zimmermann L. Matthewson, C. Meier (ed.), Wiley's semantics companion, Wiley and Sons.

Nouwen, Rick. To appear. Free choice and distribution over disjunction: the case of free choice ability. Semantics \& Pragmatics.

Renans, Agata, Jacopo Romoli, Maria-Margarita Makri, Lyn Tieu, Hanna de Vries, Raffaella Folli \& George Tsoulas. 2018. The abundance inference of pluralised mass nouns is an implicature: Evidence from Greek. Glossa 3(1). 103.

Romoli, Jacopo \& Paolo Santorio. 2019. Filtering free choice. Semantics \& Pragmatics 12. doi:10.3765/sp.12.12.

Rothschild, Daniel \& Stephen Yablo. 2018. Permissive updates. Ms., UCL and MIT.

Santorio, Paolo \& Jacopo Romoli. 2017. Probability and implicatures: A unified account of the scalar effects of disjunction under modals. Semantics \& Pragmatics 10(13). 
Skordos, Dimitrios, Roman Feiman, Alan Bale \& David Barner. 2018. Do children interpret "or" conjunctively? Ms., University of Calgary.

Starr, Will. 2016. Expressing permission. In Semantics and Linguistic Theory 26, 325-349.

van Tiel, Bob, Emiel van Miltenburg, Natalia Zevakhina \& Bart Geurts. 2016. Scalar diversity. Journal of Semantics 33(1). 137-175.

Tieu, Lyn, Cory Bill, Jacopo Romoli \& Stephen Crain. 2017a. Plurality inferences are scalar implicatures: evidence from acquisition. Ms., Maquarie University and Ulster University.

Tieu, Lyn, Manuel Kriz \& Emmanuel Chemla. 2019. Children's acquisition of homogeneity in plural definite descriptions. Frontiers in Psychology. doi:10.3389/fpsyg.2019.02329.

Tieu, Lyn, Jacopo Romoli, Peng Zhou \& Stephen Crain. 2016. Children's knowledge of free choice inferences and scalar implicatures. Journal of Semantics 33(2). 269-298.

Tieu, Lyn, Kazuko Yatsushiro, Alexandre Cremers, Jacopo Romoli, Uli Sauerland \& Emmanuel Chemla. 2017b. On the role of alternatives in the acquisition of simple and complex disjunctions in french and japanese. Journal of Semantics 34(1). 127-152. doi:10.1093/jos/ffw010.

Van Tiel, Bob. 2012. Universal free choice? In Ana Aguilar et al. Guevara (ed.), Sinn und bedeutung 16, 627-638.

Willer, Malte. 2017. Widening free choice. In The 21st Amsterdam Colloquium, 511-520.

Zimmerman, Thomas Ede. 2000. Free choice disjunction and epistemic possibility. Natural Language Semantics 8. 255-290.

Lyn Tieu

Western Sydney University

Locked Bag 1797

Penrith NSW 2751

Australia

lyn.tieu@gmail.com

Jacopo Romoli

School of Communication and Media

Ulster University, Jordanstown campus

Shore Road

Newtownabbey

Co. Antrim

BT37 0QB

j.romoli@ulster.ac.uk
Cory Bill

Leibniz-ZAS

Schützenstraße 18

10117, Berlin

Germany

corybill@gmail.com 